\title{
A Simple Method to Design and Analyze Dynamic Vibration Absorber of Pipeline Structure Using Dimensional Analysis
}

\author{
Mehrdad Shemshadi $\left(\mathbb{D},{ }^{1}\right.$ Mahdi Karimi $\mathbb{D}^{1},{ }^{1}$ and Farzad Veysi $\mathbb{D}^{2}$ \\ ${ }^{1}$ Department of Mechanical Engineering, Bu-Ali Sina University, Hamedan 65178-38695, Iran \\ ${ }^{2}$ Department of Mechanical Engineering, Razi University, Kermanshah 67144-14971, Iran \\ Correspondence should be addressed to Mahdi Karimi; m_karimi@basu.ac.ir
}

Received 2 February 2020; Revised 22 May 2020; Accepted 12 June 2020; Published 8 August 2020

Academic Editor: Fabio Rizzo

Copyright (c) 2020 Mehrdad Shemshadi et al. This is an open access article distributed under the Creative Commons Attribution License, which permits unrestricted use, distribution, and reproduction in any medium, provided the original work is properly cited.

\begin{abstract}
Vibrations due to mechanical excitation and internal and external fluid flow can cause fatigue in pipelines and leaks in fittings. A beam-based dynamic vibration absorber (beam DVA) is a device comprising an L-shaped beam with a concentrated mass at its free end that can be used to absorb and dissipate vibrations in the pipeline. In this paper, a mathematical equation is extracted to design the beam DVA using the dimensional analysis (DA) method and data recorded from 120 experimental tests. In the experimental studies, the pipes are fabricated in 1-inch, 2-inch, and 3-inch sizes. Each pipe is subjected to harmonic excitation at different frequencies, and the amplitude of vibration of the pipe is evaluated by changes in the geometric characteristics of beam DVA and concentrated mass. The proposed methodology is validated using the finite element method and simulation in the SIMULINK/MATLAB. The results showed that, out of the nine effective dimensionless parameters identified in pipe vibration control, mass ratio and stiffness ratio have the highest and lowest impacts on pipe vibration absorption, respectively.
\end{abstract}

\section{Introduction}

Pipeline structures are used in oil and gas industries, petroleum industries, power plants, and chemical factories. Due to the wide application of pipes in various industries, vulnerability assessment and improvement of piping systems and pipelines in the field of vibration are of great importance. Usually, piping systems are designed against static load cases only. However, often, dynamic loads occur. Vibration excitations in a pipe are induced by turbulent flow in the pipe, water and liquid hammers, and pressure pulsations, caused by driven machines. If the frequency of excitation is close to the natural frequency of the piping system, resonation occurs, causing material fatigue, making them prone to leaking joints, causing safety hazards in the industries, and finally leading to damage and failure. In this situation, the vibration can be reduced using a dynamic vibration absorber. Many studies have been conducted in this regard, each one with advantages and disadvantages.
Accordingly, the mathematical modeling of the vibrational behavior of the pipes and finding the optimum parameters of the vibration absorbers in the pipelines have been of interest to researchers in recent years. Norris et al. [1] worked on the application of adjustable vibration absorbers in controlling pipeline vibrations caused by wind force excitation. Although the amplitude of vibration caused by wind power is relatively small, repeated cyclic vibrations can cause fatigue in pipeline connections. The vibration absorber consists of a mass and a flexible element. This paper considers the impedance at the absorber connection point against the impedance of the primary system and the absorber system. In addition, the amplitude reduction factor of the primary structure in excitation frequency is used to design the required absorber mass. The absorber consists of two mass-held elastomeric discs. Elastomeric discs act as a rotating spring. The masses can be easily added and subtracted to adjust the absorber. The absorber is mounted on 60 different pipeline structures with $2^{\prime \prime}$ to $24^{\prime \prime}$ in diameter over more than 10 years in Northern Alaska. Nabeel et al. [2] 
investigated the vibrational and stability properties of a welding pipe. Welding in pipes causes residual stresses which affect the vibrational properties of the pipe. The Euler-Bernoulli beam theory and plug-flow model were used to model the pipe and the effects of residual stress on the vibrational properties of the pipe, respectively. The fluid velocity and pressure distribution inside the pipe were extracted using the finite element method. The results show that the natural frequencies of the welding pipe decrease with increasing fluid velocity. Welding in the fluid carrier pipe results in a decrease in the normal frequencies; changes in frequencies depend on the location of the welding. Senalp et al. [3] investigated the dynamic response of the Euler-Bernoulli beam to the linear and nonlinear viscoelastic support with respect to centralized impetus. Equations of motion were extracted using mass and stiffness matrices in Euler-Bernoulli theory, and the model was analyzed using the finite element method. Demir and Civalek [4] investigated the vibration of the nanoscaled beam embedded in an elastic matrix under the effect of thermal environments. This document describes the elemental mass and stiffness matrices via Hermitian cubic shape functions for the nanoscaled beam. The influences of the Pasternak foundation parameter, small scale parameter, and mechanical properties of material and thermal effect on vibrational frequency are investigated. The results show that the difference between the frequencies of classical beam model and those of the nonlocal beam model increases as the mode number and nonlocal parameter increase. Lats [5] studied the vibrations of a beam connected to a rotational transient vibrational absorber system. The beam was subjected to harmonic and central excitation forces; the Euler-Bernoulli beam theory was used for modeling. The Fourier method was applied to analyze the model, and the displacement amplitude of the beam arbitrary points at the desired frequencies was obtained using the Laplace transform. This article aims to investigate the performance of a translational-rotational absorber compared with a translational absorber. Numerical simulations show a significant improvement in vibration reduction when using translational-rotational absorbers. Civalek [6] derived the frequencies and buckling loads of composite plates using the discrete singular convolution (DSC). This method is a good level of accuracy dynamic analysis method for single structures. However, there are also limitations. In this study, the straight-sided quadrilateral domain is mapped into natural coordinates, and the frequencies and buckling loads are accurately predicted. The results are compared with different numerical methods. Syaiful et al. [7] presented a simple support beam theory model with dynamic mass absorber, spring, and damper. The Euler-Bernoulli theory was applied to obtain the displacement amplitude of the beam from the governing equations, and the beam displacement equations were obtained with the absorber using the receptance theory at the beam connection point. In addition, the governing equations of the absorber were extracted using equations of motion and calculated according to the boundary conditions of the displacement to force ratio. MATLAB was used to draw the frequency response function (FRF) diagrams, and ANSYS was used to validate the results. A comparison of theoretical solutions and drawing results with MATLAB and simulation with ANSYS revealed a small error of about $0.1 \%$. The results show that the displacement amplitude decreases by about $30 \%$ with increasing vibration absorber to the beam. Zainulabidin et al. [8] investigated the effect of vibration absorbers mounted on beams with clamped support. The spring-mass vibration absorber was mounted on four beams with clamped support and analyzed using ANSYS. First, the natural frequencies and mode shapes in the beam were measured using modal analysis; then, the effect of the location of the absorbers on the beam was studied in terms of vibrational properties. The results show that the location of the absorber strongly influences the amplitude of the vibration of the beam. Also, a high percentage of amplitude reduction is obtained when absorbers are not located on the node. When the vibration absorber is near the fixed supports, the amplitude of vibration decreases to $9.99 \%$ and $99.8 \%$ in the first and second modes, respectively. Sutar et al. [9] developed a mathematical model for dynamics and stability of the pipe conveying the fluid with guided supports and compared it with the finite element method (FEM). The Euler-Bernoulli theory and Hamilton's energy expressions were applied to obtain the equation of motion and fundamental transverse natural frequency of vibration. The influence of fluid velocity on natural frequencies was extracted using the ABAQUS software for three different pipe support materials: thermocol, foam, and springs with a stiffness of $54 \mathrm{~N} / \mathrm{mm}$. The results show that, for foam type of support, the frequencies tend to vary with increase in flow velocities, and for the other type of supports, it is seen that the frequencies reduce for the fluid flow condition only when compared with the other two conditions. Song et al. [10] investigated the vibration control of a pipeline using a pounding tuned mass damper (PTMD) absorber. In this study, an M-shaped pipeline was constructed with the PTMD absorber mounted on it. Due to the lack of empirical studies on the fluid carrier pipe because of safety and operational issues in the working industries, the pipe was considered as free and fluid-independent; also, the fluid effects on the vibrational properties of the pipe were ignored. The PTMD absorber consists of two parts: the absorber part and the separator part. The vibration absorber part consists of a cantilever beam, with a concentrated mass in the free head, mounted horizontally and parallel to the pipe axis. The separator part is a sheet from which a circular hole is cut, the inner surface of which is covered with a viscoelastic material. When the mass fluctuations exceed a certain level, the beam bends and hits the viscoelastic material. As a result, it depletes the kinetic energy and reduces the pipeline vibration. The pipeline modeling is performed using FEM as well as mass and stiffness matrices. The Rayleigh model is used to obtain the attenuation matrix, and the Hertz model is used to obtain the nonlinear shock force model in viscoelastic materials. Since the response of the system under other stimuli (e.g., vortex fluid flow and internal and external fluid flows) may be very different from the response under harmonic excitation, harmonic excitation was considered in 
this study. Experimental results show the high effectiveness of PTMD in reducing vibration. Steady-state displacement and pipe acceleration responses decreased by $86.6 \%$ and $83.6 \%$, respectively. An acceptable concordance was obtained by comparing the numerical results with the experimental data. Fong and Mohd Yassin [11] investigated the vibrational properties of oil-bearing pipelines considering the effects of fluid inside the pipe. In this paper, FEM was applied to calculate the natural frequency of a fluid carrier pipeline located on a viscoelastic foundation. The simple support is modeled using the Euler-Bernoulli beam theory. In addition, mass, stiffness, and damping matrices were extracted using the Galerkin weighted residual method. The results show that an increase in the foundation stiffness causes an increase in the natural frequency of the pipeline, while an increase in the foundation damping and fluid velocity inside the pipe causes a decrease in the natural frequency of the pipeline. The results were validated using the data from the previous studies. Chang et al. [12] investigated the vibration control of a nuclear power plant's piping system using an earthquake Stockbridge damper. The piping system analysis was performed using SAP2000, both with and without a damper. The damper design was performed in two stages: (1) calculation of mass ratio, frequency ratio, and attenuation ratio and (2) absorber design. The best damper design parameters were extracted according to the fixed-point method. The results show that using vibration absorbers can be effective in controlling the vibration response of the piping system in the nuclear industry. Hua et al. [13] examined the model of a cantilever beam as the primary structure by connecting another cantilever beam at its end as a dynamic vibration absorber. The combination of receptance and fixed-point methods was used to optimally extract the vibration absorber characteristics. The analytical results were validated using ABACUS. Finally, a comparison was made between beams as vibration absorbers and traditional spring damper absorbers. The optimum mass ratio and frequency ratio in the traditional absorber was obtained using the fixed-point method. Also, the maximum amplitude was calculated at two points. The results show that the maximum amplitude is lower when the beam is used as vibration absorbers. Tyng et al. [14] performed a study on a laboratory-scale piping system to investigate the flow-induced vibration problems under different operating conditions. The study of fluid flow-induced vibrations in pipes in real piping systems at operating sites raises safety and operational concerns; therefore, a laboratory-scale piping system is preferred to investigate the flow-induced vibration problem under various operating conditions. A smaller-scale model employs dimensional analysis as well as geometric, kinematic, and dynamic similarities to obtain reliable results. In the geometric similarity, both real and scaled models must have the same length to diameter ratio, as required by Buckingham's theorem. In addition, the mode shape of the scaled model must be like that of the real model in natural frequency. The flow pattern of the models can be used to justify the kinematic similarity. This pattern is similar when the Reynolds number in the real and scalable model is the same. Real and scaled Reynolds numbers are derived from computational fluid dynamics (CFD) simulation results. Fluid-structure interaction (FSI) or CFD analysis should be performed to investigate the dynamic similarity. When the force properties of the fluid and the wall of the pipe are similar, it can be concluded that the dynamic similarity is obtained. The geometric, kinematic, and dynamic similarities were obtained in a case study between the scaled and real models.

The dimensional analysis is one of the powerful tools in engineering that enables the rapid analysis of physical systems. Although dimensional analysis is traditionally used in fluid mechanics, this method is useful in all physical and engineering sciences. Moreover, its prominent applications are found in the social sciences and biology. The idea of experimenting with unrealistic dimensions has been suggested by several individuals. Newton was one of those scientists who defined the term "principles of similarity". Later, Maxwell, a Scottish physicist, played an important role in establishing the basic units of mass, length, and time that are used in the creation of other units. Rayleigh was probably the first who applied dimensional analysis to find relationships between physical quantities. In addition, he was the first who used dimensionless parameters to analyze experimental data. The turning point of the dimensional analysis was made by Buckingham, who expressed dimensional analysis and rules of similarity more systematically. Reddy and Reddy [15] investigated different properties of ball-bearing parameters using dimensional analysis. In this study, a dimensional analysis was performed using Buckingham's Theorem. For this purpose, 20 dimensionless quantities in ball bearings were identified, and 13 dimensionless terms affecting ball bearings performance were extracted. Ball-bearing length-to-diameter (L/D) ratio diagrams were plotted in each dimensionless quantity at different values of convective heat transfer coefficients. This method can be used to find the effect of slight changes in the convective heat transfer coefficients on ball bearings. Allamraju and Srikanth [16] extracted the equations governing the PZT-5H transducer using Buckingham's Theorem. Hassani et al. [17] developed new equations to predict the thermal conductivity of nanofluid based on experimental data using Buckingham's Theorem. This author developed a new equation that can predict thermal conductivity for a range of nanofluids. The thermal conductivity of nanofluids depends on the base fluid, temperature, thermal conductivity, viscosity, density, or physical properties of the nanoparticles. Thakare et al. [18] extracted a mathematical model to predict the displacement of the upper roller of the 3 -roll bending machine using dimensional analysis. They used valid experimental data in the developed model and obtained the error rate of $1.237 \%$ for the upper roller displacement, suggesting that the values obtained from the mathematical model are close to the actual values in the experimental tests.

One of the most important issues in using vibration absorbers is the optimal design of their parameters. If the vibration absorber parameters are inappropriate, the existence of the absorber could even cause an increase in the amplitude of vibrations. In this paper, the authors propose 
the use of experimental tests and dimensional analysis to optimally design beam DVA in the piping systems. The use of Buckingham's theorem allows the identification of effective and less effective dimensionless numbers involved in the amplitude of vibration of the pipe and the derivation of functional, simple, and reliable equations in system analysis.

\section{Experimental Model}

According to Figures 1 and 2, the experimental model consists of four parts: pipeline structure, motor with unbalanced weight, vibrometer, and beam DVA. The pipes are mounted on two T-post supports that behave like simple support. A noteworthy point in this regard is that it is not possible to carry out practical tests on operating units on fluid carrier pipelines, the necessity of changing process conditions in the system, and the need for a series of measurements and safety concerns. Hence, theoretical studies and experimental tests are performed considering a fluid-free pipe with harmonic force excitation, which may be due to mechanical excitation. In the experiments, three different sizes of pipe 1-inch, 2-inch, and 3-inch are used. Table 1 shows the geometric characteristics and physical properties of the pipeline structure used in the experimental model. The second part is the experimental model of motor with unbalanced weight, which is used for excitation. Harmonic force is applied to the pipe by a rigid rod as a result of motor. The motor is connected to a variable-frequency drive (i.e., an inverter) to allow the desired frequency to be applied. On the velocity control unit, the motor frequency can be increased or decreased by adjusting the screws. The third part is the experimental vibrometer model. A portable vibrometer with a frequency range of 2 to $10,000 \mathrm{~Hz}$ is used to measure the frequency, acceleration, velocity, and displacement as well as drawing an FFT diagram. The fourth part is the experimental beam DVA model. This device consists of an L-shaped beam, with a concentrated mass added to its end, which can move in both horizontal and vertical directions and absorb kinetic energy. The use of threaded rod types in beam construction provides high flexibility in design. This flexibility is because of the ability to adjust its length under varying conditions and to modify cross-sectional dimensions. Table 2 illustrates the geometric characteristics and physical properties of the threaded rods used. The beam DVA is installed in the middle of the pipe with the most displacement, and the tests are performed with or without the beam DVA.

As illustrated in Figure 2, the experimental setup consist of a motor (brand: Kem-P, model: EVM 65/3), inverter (brand: LS, model: SV004iE5-1), accelerometer (brand: CTC, model: AC102-1A), and vibrometer (brand: KOHTec, model:107B).

Each pipe is subjected to harmonic excitation at different frequencies. Moreover, the amplitude of vibration of the pipe is recorded and evaluated by changes in geometric characteristics of beam DVA and concentrated mass. Finally, 120 experiments were performed in three pipe sizes:

Forty items in a $1^{\prime \prime}$ pipe in an excitation frequency range of $8-12.5 \mathrm{~Hz}$, concentrated mass range of $0.8-5 \mathrm{~kg}$, and threaded rods M6, M8, M10, and M12. Forty items in a $2^{\prime \prime}$ pipe in an excitation frequency range of $10-18.5 \mathrm{~Hz}$, concentrated mass range of $2.5-10 \mathrm{~kg}$, and threaded rods $\mathrm{M} 6$, M8, M10, and M12. Forty items in a $3^{\prime \prime}$ pipe in an excitation frequency range of $17-25 \mathrm{~Hz}$, concentrated mass range of $2.5-14.5 \mathrm{~kg}$, and threaded rods M8, M10, and M12.

\section{Determination of Natural Frequencies}

To derive the natural frequencies of the pipe using the Euler-Bernoulli beam theory, first, the equations of motion of the transverse pipe vibrations are extracted as partial differential equations (PDEs) and the boundary conditions governing simple support are applied. The natural frequencies are obtained by solving the differential equations by the separation of variables. The first two natural frequencies of $1^{\prime \prime}, 2^{\prime \prime}$, and $3^{\prime \prime}$ pipes with the specifications listed in Table 1 are $9.43(37.72) \mathrm{Hz}, 17.02(68.08) \mathrm{Hz}$, and $25.40(101.60) \mathrm{Hz}$, respectively. The first mode has the lowest frequency and the largest displacement. Therefore, this paper studies the first vibration mode, which is also the most important one.

To extract the natural frequency of the pipe in experimental tests, first, the frequency of the vibrator motor is slowly increased by using the inverter to cause resonance in the system to obtain the natural frequency of the system. Using the data recorded by the accelerometer, the first natural frequency for the $1^{\prime \prime}, 2^{\prime \prime}$, and $3^{\prime \prime}$ pipes are 10.02, 16.9, and $23.7 \mathrm{~Hz}$, respectively. A comparison of the results shows that the estimation of the first natural frequency of the pipe is very well approximated in the experimental tests, which proves the accuracy and efficiency of the experimental tests. Figure 3 shows the comparison of the $1^{\prime \prime}$ pipe FRF diagram in theory and the data recorded by the vibrometer.

\section{Formulation of Mathematical Model Using DA}

Several parameters affect the performance of the beam DVA, which can lead to the complexity of problem analysis and design. As a very powerful tool, dimensional analysis eliminates the complexity and reduces the number of effective variables by compressing some parameters. For this purpose, it is necessary to determine the parameters affecting the performance of the system and reducing the vibrations, which are of high importance. All the variables affecting this phenomenon are

$$
f\left(x_{p}, x_{p s}, L_{a}, L_{p}, M_{a}, w, I_{a}, I_{p}, A_{a}, A_{p}, E_{a}, E_{p}, \rho_{a}, \rho_{p}\right)=0 .
$$

Determining the function of equation (1) requires a great deal of time and many experimental tests. Using dimensional analysis, this phenomenon can be formulated using an equation between only a few dimensionless groups. Each of these dimensionless groups is called $\pi$. Now, the question arises as to how many $\pi$ can be formed for this number of variables known to be effective in the beam DVA. The Buckingham $\pi$ theorem is used to respond to this question. According to this theory, the number of the independent 


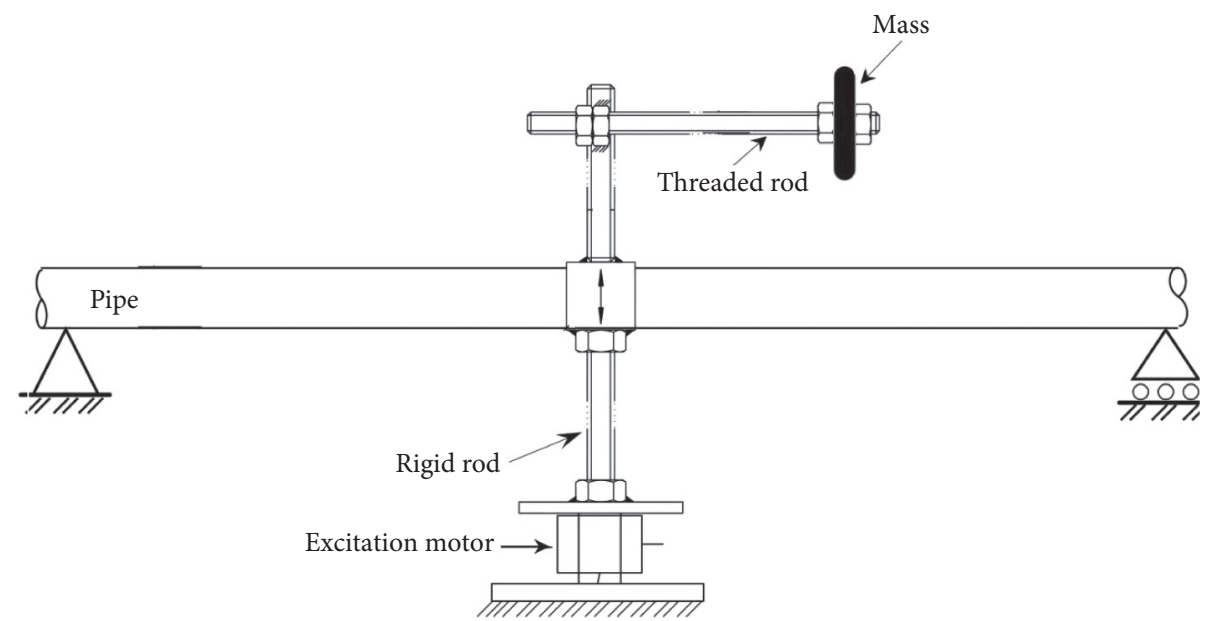

Figure 1: Schematic of the pipeline structure and beam DVA.



Figure 2: Picture of the experimental setup.

TABle 1: Parameters of the pipeline structure.

\begin{tabular}{lcccc}
\hline & $\begin{array}{c}\text { Carbon steel pipe seamless, density }=7850 \mathrm{~kg} / \mathrm{m}^{3} \text {, span, Young's modulus }=210 \mathrm{GPa} \text {, according to API5LGr.A } \\
\text { NPS }\end{array}$ & Wall thickness $(\mathrm{mm})$ & \multicolumn{1}{c}{ Weight $(\mathrm{kg})$} \\
\hline 1 inch & Outside diameter $(\mathrm{mm})$ & 0.00338 & 3.032 & 7.58 \\
2 inches & 0.0334 & 0.00391 & 3.087 & 16.79 \\
3 inches & 0.0603 & 0.00396 & 3.10 & 25.69 \\
\hline
\end{tabular}

TABle 2: Parameters of the L-beam.

\begin{tabular}{lcccr}
\hline & \multicolumn{2}{c}{ Threaded rod, $1 \mathrm{~m}$, density $=7850 \mathrm{~kg} / \mathrm{m}^{3}$, Young's modulus $=200 \mathrm{GPa}$, according to ISO 724} \\
Steel & Pitch $(\mathrm{mm})$ & Pitch diameter $(\mathrm{mm})$ & Minor diameter $(\mathrm{mm})$ & Property class $(\mathrm{DIN}$ 976) \\
\hline M6 & 1 & 5.350 & 4.917 & 5.6 \\
M8 & 1.25 & 7.188 & 6.647 & 5.6 \\
M10 & 1.5 & 9.026 & 8.376 & 5.6 \\
M12 & 1.5 & 11.026 & 10.376 & 5.6 \\
M16 & 1.5 & 15.026 & 14.376 & 5.6 \\
\hline
\end{tabular}




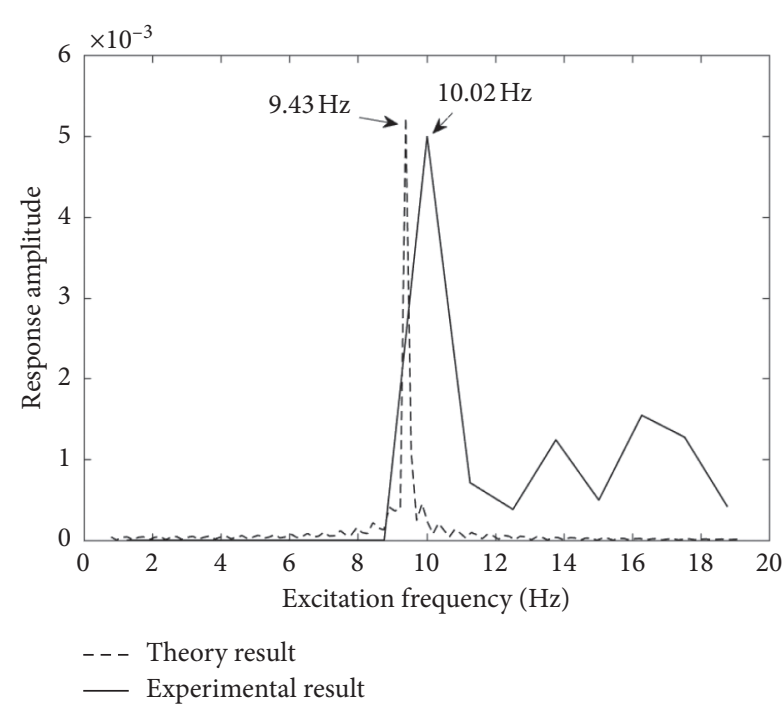

FIgURE 3: Comparison between theoretical and experimental results (first mode, 1-inch pipeline).

dimensionless groups that can be used to describe a phenomenon is $n-r$, where $n$ is the number of the variables influencing the phenomenon and $r$ is the number of the main dimensions required for dimensional. Since the values of modulus of elasticity and density in the pipe and beam DVA are very close to each other in experimental tests, 10 parameters are selected from the 14 effective parameters, where there are 7 independent groups $\pi$ and one dependent group $\pi$. Tables 3 and 4 represent the parameters along with the main $M$, $\mathrm{L}$, and $T$ quantities.

Each dependent $\pi_{i}$ term is a function of independent $\pi_{i}$ where the amplitude ratio of the pipeline $\pi_{S}$ can be shown by dimensionless parameters as

$$
\frac{x_{\mathrm{pa}}}{x_{p}}=K\left(\frac{M_{a}}{M_{p}}\right)^{a_{1}}\left(\frac{L_{a}}{L_{p}}\right)^{a_{2}}\left(\frac{w}{w_{\mathrm{na}}}\right)^{a_{3}}\left(\frac{w_{\mathrm{na}}}{w_{\mathrm{np}}}\right)^{a_{4}}\left(\frac{K_{a}}{K_{p}}\right)^{a_{5}}\left(\frac{I_{a}}{I_{p}}\right)^{a_{6}}\left(\frac{A_{a}}{A_{p}}\right)^{a_{7}} .
$$

That could be expressed in the following form:

$$
\pi_{S}=f\left(\pi_{1}, \pi_{2}, \pi_{3}, \pi_{4}, \pi_{5}, \pi_{6}, \pi_{7}\right) .
$$

A probable exact mathematical form for the dimensional equations of the phenomenon could be assumed to be of the exponential form as follows:

$$
\pi_{S}=k_{1} \times \pi_{1}^{a_{1}} \times \pi_{2}^{a_{2}} \times \pi_{3}^{a_{3}} \times \pi_{4}^{a_{4}} \times \pi_{5}^{a_{5}} \times \pi_{6}^{a_{6}} \times \pi_{7}^{a_{7}} .
$$

where $a_{1}-a_{7}$ and curve constant $k_{1}$ are derived from data collected from experimental tests. First, equation (4) is transformed into the logarithmic form as follows:

$$
\begin{aligned}
\log \pi_{S}= & \log k_{1}+a_{1} \log \pi_{1}+a_{2} \log \pi_{2}+a_{3} \log \pi_{3}+a_{4} \log \pi_{4} \\
& +a_{5} \log \pi_{5}+a_{6} \log \pi_{6}+a_{7} \log \pi_{7},
\end{aligned}
$$

where $\quad Z=\log \pi_{S}, \quad K=\log k_{1}, \quad A=\log \pi_{1}, \quad B=\log \pi_{2}$, $C=\log \pi_{3}, \quad D=\log \pi_{4}, \quad E=\log \pi_{5}, \quad F=\log k_{6}, \quad$ and $G=$ $\log \pi_{7}$.

Putting the values in equation (5), the same can be written as

$$
Z=K+a_{1} A+a_{2} B+a_{3} C+a_{4} D+a_{5} E+a_{6} F+a_{7} G .
$$

In this step, regression analysis is utilized. equation (6) is a regression equation of $Z$ in $A, B, C, D, E, F$, and $G$. By multiplying equation (6) by $A, B, C, D, E, F$, and $G$, separately, a set of simultaneous equations is obtained:

$$
\begin{aligned}
\sum Z & =N \times K+a_{1} \sum A+a_{2} \sum B+a_{3} \sum C+a_{4} \sum D+a_{5} \sum E+a_{6} \sum F+a_{7} \sum G \\
\sum Z A & =K \sum A+a_{1} \sum A A+a_{2} \sum A B+a_{3} \sum A C+a_{4} \sum A D+a_{5} \sum A E+a_{6} \sum A F+a_{7} \sum A G \\
\sum Z B & =K \sum B+a_{1} \sum B A+a_{2} \sum B B+a_{3} \sum B C+a_{4} \sum B D+a_{5} \sum B E+a_{6} \sum B F+a_{7} \sum B G \\
\sum Z C & =K \sum C+a_{1} \sum C A+a_{2} \sum C B+a_{3} \sum C C+a_{4} \sum C D+a_{5} \sum C E+a_{6} \sum C F+a_{7} \sum C G \\
\sum Z D & =K \sum D+a_{1} \sum D A+a_{2} \sum D B+a_{3} \sum D C+a_{4} \sum D D+a_{5} \sum D E+a_{6} \sum D F+a_{7} \sum D G \\
\sum Z E & =K \sum E+a_{1} \sum E A+a_{2} \sum E B+a_{3} \sum E C+a_{4} \sum E D+a_{5} \sum E E+a_{6} \sum E F+a_{7} \sum E G \\
\sum Z F & =K \sum F+a_{1} \sum F A+a_{2} \sum F B+a_{3} \sum F C+a_{4} \sum F D+a_{5} \sum F E+a_{6} \sum F F+a_{7} \sum F G \\
\sum Z G & =K \sum G+a_{1} \sum G A+a_{2} \sum G B+a_{3} \sum G C+a_{4} \sum G D+a_{5} \sum G E+a_{6} \sum G F+a_{7} \sum G G .
\end{aligned}
$$


TABLE 3: List of variables and fundamental dimensions that affect the beam DVA attached to the pipeline structure.

\begin{tabular}{lcccccc}
\hline No. & Description of variables & Symbol & Unit & Dimensions & Type of variable & Nature \\
\hline 1 & Span & $L_{p}$ & $\mathrm{~m}$ & {$\left[M^{0} L^{1} T^{0}\right]$} & Independent & Constant \\
2 & Length of the threaded rod & $L_{a}$ & $\mathrm{~m}$ & {$\left[M^{0} L^{1} T^{0}\right]$} & Independent & Varying \\
3 & Mass of the beam DVA & $M_{a}$ & $\mathrm{~kg}$ & {$\left[M^{1} L^{0} T^{0}\right]$} & Independent & Varying \\
4 & Moment of inertia of the pipe & $I_{p}$ & $\mathrm{~m}^{4}$ & {$\left[M^{0} L^{4} T^{0}\right]$} & Independent & Constant \\
5 & Moment of inertia of the threaded rod & $I_{a}$ & $\mathrm{~m}^{4}$ & {$\left[M^{0} L^{4} T^{0}\right]$} & Independent & Varying \\
6 & Area of the pipe & $A_{p}$ & $\mathrm{~m}^{2}$ & {$\left[M^{0} L^{2} T^{0}\right]$} & Independent & Constant \\
7 & Area of the threaded rod & $A_{a}$ & $\mathrm{~m}^{2}$ & {$\left[M^{0} L^{2} T^{0}\right]$} & Independent & Varying \\
8 & Modulus of elasticity of the pipe & $E_{p}$ & $\mathrm{~N} / \mathrm{m}^{2}$ & {$\left[M^{1} L^{-1} T^{-2}\right]$} & Independent & Constant \\
9 & Modulus of elasticity of the threaded rod & $E_{a}$ & $\mathrm{~N} / \mathrm{m}^{2}$ & {$\left[M^{1} L^{-1} T^{-2}\right]$} & Independent & Constant \\
10 & Density of the pipe & $\rho_{p}$ & $\mathrm{~kg} / \mathrm{m}^{3}$ & {$\left[M^{1} L^{-3} T^{0}\right]$} & Independent & Constant \\
11 & Density of the threaded rod & $\rho_{a}$ & $\mathrm{~kg} / \mathrm{m}^{3}$ & {$\left[M^{1} L^{-3} T^{0}\right]$} & Independent & Constant \\
12 & Frequency of exciting force & $w$ & $\mathrm{rad} / \mathrm{s}$ & {$\left[M^{0} L^{0} T^{-1}\right]$} & Independent & Varying \\
13 & Displacement at the middle of the pipe without beam DVA & $x_{p}$ & $\mathrm{~m}$ & {$\left[M^{0} L^{1} T^{0}\right]$} & Dependent & Response \\
14 & Displacement at the middle of the pipe with beam DVA & $x_{p a}$ & $\mathrm{~m}$ & {$\left[M^{0} L^{1} T^{0}\right]$} & Dependent & Response \\
\hline
\end{tabular}

TABLE 4: List of $\pi_{i}$ groups of the beam DVA attached to the pipeline structure.

\begin{tabular}{lc}
\hline No. & Dimensionless groups $\left(\pi_{i}\right.$ terms $)$ \\
\hline 1 & $\pi_{S}=\left(x_{\mathrm{pa}} / x_{p}\right)$ \\
2 & $\pi_{1}=\left(M_{a} / M_{p}\right)$ \\
3 & $\pi_{2}=\left(L_{a} / L_{p}\right)$ \\
4 & $\pi_{3}=\left(w / w_{n a}\right)$ \\
5 & $\pi_{4}=\left(w_{\mathrm{na}} / w_{\mathrm{np}}\right)$ \\
6 & $\pi_{5}=\left(K_{a} / K_{p}\right)$ \\
7 & $\pi_{6}=\left(I_{a} / I_{p}\right)$ \\
8 & $\pi_{7}=\left(A_{a} / A_{p}\right)$ \\
\hline
\end{tabular}

From the data recorded from 120 experimental tests, the unknown coefficients of equation (6) can be derived by Cramer's rule in MATLAB by replacing the derived unknown coefficients in equation (6), which expresses the correlation between the amplitude ratio of the pipeline without and with the beam DVA $\left(\pi_{S}\right)$ and other dimensionless parameters.

$$
\begin{aligned}
\pi_{S}= & 0.44668 \times \pi_{1}^{0.7684} \times \pi_{2}^{-0.744} \times \pi_{3}^{-1.1352} \times \pi_{4}^{0.6319} \times \pi_{5}^{-1.3772} \\
& \times \pi_{6}^{0.5096} \times \pi_{7}^{-0.6847} .
\end{aligned}
$$

This correlation represents a new correlation of the amplitude ratio $\pi_{S}$ as a function of other dimensionless parameters $\pi$ of the system.

The coefficient of determination $R^{2}$ is used to estimate the accuracy between the values of equation (8) and experimental results. The following diagram in Figure 4 shows a comparison between the values of the experimental test data $\left(x_{\mathrm{pa}} / x_{p}\right)$ and the calculated $\pi_{S}$.

To identify the compact and simpler correlations that can help design and tuning beam DVA for pipeline structures, the above steps are considered with other dimensionless parameters $\pi$ and the unknown coefficients are calculated.

The collection of sample data used to establish the amplitude ratio $\pi_{S}$ by $\pi_{i}$ terms and compare it with experimental results $\left(x_{\mathrm{pa}} / x_{p}\right)$ is shown in Table 5 .

4.1. Indices of $\pi_{\mathbf{i}}$ Terms for $\pi_{\mathrm{S}}$. In this section, according to Equation (8), the effects of dimensionless parameters on amplitude ratio $\pi_{S}$ of pipeline structures are discussed. As it is indicated in Figure 5, $\pi_{1}$ has the greatest effect on amplitude ratio.

(i) $\pi_{1}$ is 0.7684 , which is the greatest. Thus, $\pi_{1}$ has the greatest effect on amplitude ratio. The positive value shows that $\pi_{1}$ has a direct relationship with $\pi_{S}$.

(ii) $\pi_{5}$ is -1.3772 , which is the lowest. Thus, $\pi_{5}$ has the lowest effect on ratio amplitude. The negative value demonstrates that $\pi_{5}$ has an inverse relationship with $\pi_{S}$.

(iii) The curve constant in the model is 0.44668 , which shows the effects of other factors on ratio amplitude.

\section{Numerical Model}

The finite element (FE) model is used to validate the methodology presented. First, the equations governing the motion of the pipeline are extracted using the Euler-Bernoulli beam element, as shown in Figure 6. The nodes are under the displacement and rotation of $w_{1}(t), w_{2}(t), w_{3}(t)$, and $w_{4}(t)$. The forces $f_{1}(t)$ and $f_{3}(t)$ are proportional to the displacements of $w_{1}(t)$ and $w_{3}(t)$, and the moments $f_{2}(t)$ and $f_{4}(t)$ are proportional to the rotations of $w_{2}(t)$ and $w_{4}(t)$, respectively. The transverse beam displacement is assumed to be proportional to a cubic equation. After obtaining the shape functions using kinetic energy, the bending strain energy and the virtual work of the mass and stiffness matrices are obtained as follows:

$$
\begin{gathered}
{[m]=\frac{\rho A L}{420}\left[\begin{array}{cccc}
156 & 22 l & 54 & -13 l \\
22 l & 4 l^{2} & 13 l & -3 l^{2} \\
54 & 13 l & 156 & -22 l \\
-13 l & -3 l^{2} & -22 l & 4 l^{2}
\end{array}\right],} \\
{[k]=\frac{\mathrm{EI}}{L^{3}}\left[\begin{array}{cccc}
12 & 6 l & -12 & 6 l \\
6 l & 4 l^{2} & -6 l & 2 l^{2} \\
-12 & -6 l & 12 & -6 l \\
6 l & 2 l^{2} & -6 l & 4 l^{2}
\end{array}\right] .}
\end{gathered}
$$




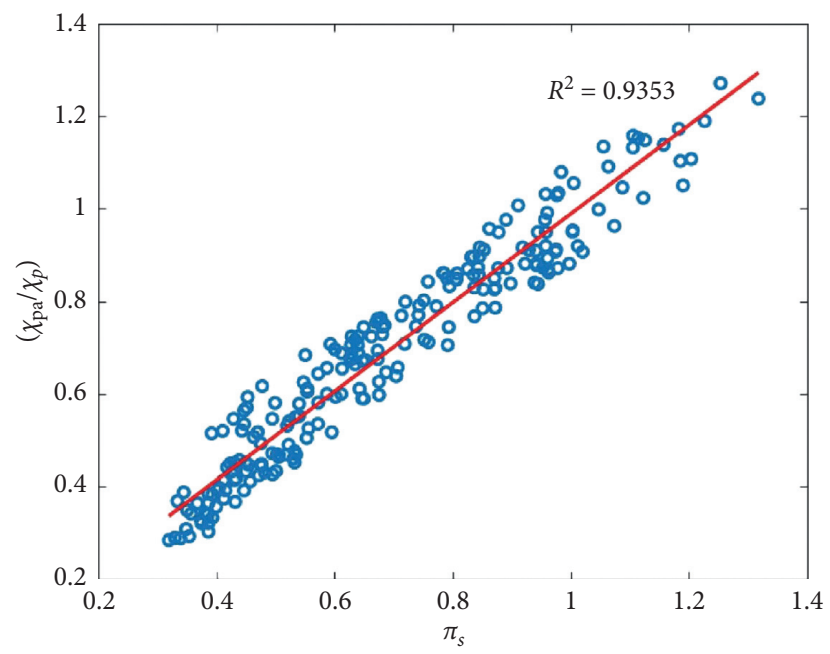

FIgURE 4: Comparison of the experimental data $\left(x_{\mathrm{pa}} / x_{p}\right)$ with $\pi_{S}$.

TABLE 5: Comparison of amplitude ratio $\pi_{S}$ results vs. experimental results $\left(x_{\mathrm{pa}} / x_{p}\right)$.

\begin{tabular}{|c|c|c|c|c|c|c|c|c|}
\hline No. & NPS pipe (inch) & Excited frequency $(\mathrm{Hz})$ & $\left(x_{\mathrm{pa}} / x_{p}\right)$ & Size pitch & \multicolumn{2}{|c|}{$\mathrm{M}$ absorber $(\mathrm{kg})$} & \multicolumn{2}{|c|}{$\mathrm{L}$ absorber $(\mathrm{m})$} \\
\hline 1 & 1 & 9 & 0.74527 & M8 & \multicolumn{2}{|c|}{2.35} & \multicolumn{2}{|c|}{0.234} \\
\hline 2 & 1 & 9.5 & 0.94950 & M6 & \multicolumn{2}{|c|}{0.8} & \multicolumn{2}{|c|}{0.185} \\
\hline 3 & 1 & 10 & 0.68628 & M8 & \multicolumn{2}{|c|}{1.7} & \multicolumn{2}{|c|}{0.238} \\
\hline 4 & 1 & 11 & 0.49483 & M8 & \multicolumn{2}{|c|}{2.35} & \multicolumn{2}{|c|}{0.18} \\
\hline 5 & 2 & 10 & 0.79916 & M16 & \multicolumn{2}{|c|}{10} & \multicolumn{2}{|c|}{0.342} \\
\hline 6 & 2 & 14 & 0.64739 & M10 & \multicolumn{2}{|c|}{10} & \multicolumn{2}{|c|}{0.138} \\
\hline 7 & 2 & 16.9 & 0.59943 & M8 & \multicolumn{2}{|c|}{4} & \multicolumn{2}{|c|}{0.116} \\
\hline 8 & 2 & 17.5 & 0.71376 & M6 & \multicolumn{2}{|c|}{10} & \multicolumn{2}{|c|}{0.078} \\
\hline 9 & 3 & 21 & 0.51961 & M16 & \multicolumn{2}{|c|}{14.3} & \multicolumn{2}{|c|}{0.176} \\
\hline 10 & 3 & 22 & 0.49901 & M16 & \multicolumn{2}{|c|}{10} & \multicolumn{2}{|c|}{0.19} \\
\hline 11 & 3 & 23.7 & 0.89811 & M8 & \multicolumn{2}{|c|}{2.35} & \multicolumn{2}{|c|}{0.111} \\
\hline 12 & 3 & 25 & 0.95663 & M10 & \multicolumn{2}{|c|}{2.35} & \multicolumn{2}{|c|}{0.157} \\
\hline No. & $\pi_{1}$ & $\pi_{2}$ & $\pi_{3}$ & $\pi_{4}$ & $\pi_{5}$ & $\pi_{6}$ & $\pi_{7}$ & $\pi_{S}$ \\
\hline 1 & 0.31002 & 0.07717 & 1.15798 & 0.82381 & 0.42594 & 0.00328 & 0.12162 & 0.680855 \\
\hline 2 & 0.10554 & 0.06101 & 0.97971 & 1.02781 & 0.22570 & 0.00086 & 0.06223 & 0.944129 \\
\hline 3 & 0.22427 & 0.07849 & 1.12251 & 0.94427 & 0.40482 & 0.00328 & 0.12162 & 0.634933 \\
\hline 4 & 0.31002 & 0.05936 & 0.95485 & 1.22108 & 0.93580 & 0.00328 & 0.12162 & 0.446833 \\
\hline 5 & 0.59547 & 0.11078 & 1.02535 & 0.57274 & 0.39594 & 0.00904 & 0.25600 & 0.871955 \\
\hline 6 & 0.59546 & 0.04470 & 1.09709 & 0.80623 & 0.78467 & 0.00117 & 0.09237 & 0.593110 \\
\hline 7 & 0.23819 & 0.03757 & 0.99016 & 1.00228 & 0.48506 & 0.00043 & 0.05597 & 0.649526 \\
\hline 8 & 0.59547 & 0.02526 & 1.74684 & 0.58829 & 0.41778 & 0.00011 & 0.02864 & 0.649377 \\
\hline 9 & 0.55644 & 0.05677 & 0.95058 & 0.86966 & 0.85220 & 0.00262 & 0.16781 & 0.477241 \\
\hline 10 & 0.38912 & 0.06129 & 0.93408 & 0.92716 & 0.67736 & 0.00262 & 0.16781 & 0.499099 \\
\hline 11 & 0.09144 & 0.03580 & 0.99625 & 0.93648 & 0.16239 & 0.00015 & 0.03669 & 0.98380 \\
\hline 12 & 0.09144 & 0.05064 & 1.07115 & 0.91873 & 0.15631 & 0.00034 & 0.06055 & 0.862021 \\
\hline
\end{tabular}

5.1. Equation of Motion of Pipe-Beam DVA System. Figure 7 shows a schematic of the finite element model of a beam DVA installing pipeline structure containing 10 nodes and 8 elements. In the absorber-free model, the number of nodes and elements is considered to be 7 and 6 , respectively. The mass $M_{s}$ and stiffness $K_{s}$ matrices of the system are extracted by combining the mass and stiffness matrices of each element. It should be noted that damping is neglected because of its insignificance.

The equations of motion of the pipe-beam DVA system can be written as

$$
\left[M_{s}\right] \ddot{w}+\left[K_{s}\right] w=f(t) .
$$

The mass and stiffness matrices of the pipeline are of the order of 12 with no vibration absorber and of the order of 16 with the installation of beam DVA in the piping system. Therefore, 12 equations and 16 coupled equations are extracted, respectively. The force vectors are obtained by shape coefficients.

Simulink provides the ability to analyze the system response well by changing the input parameters. The simulation consists of two parts. The first part is the simulation of 


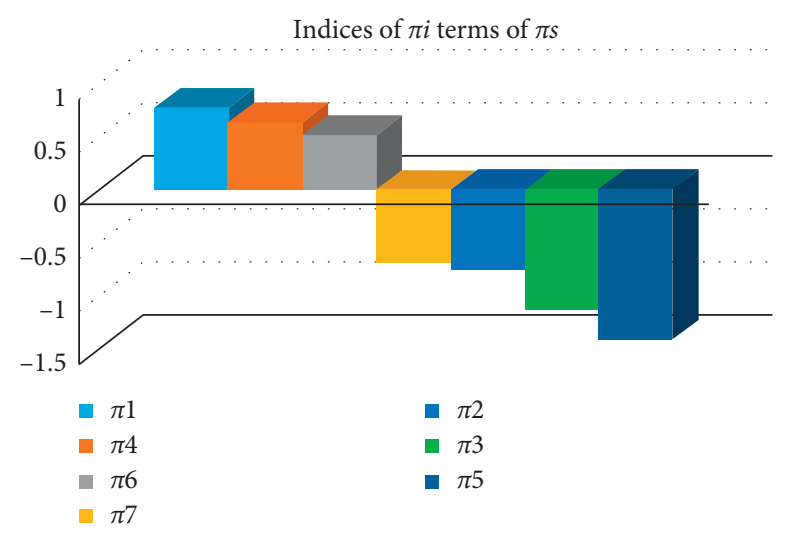

Figure 5: Indices of terms for $\pi_{S}$, according to equation (1) [8].

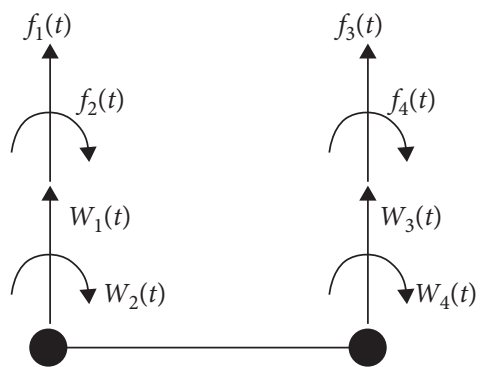

Figure 6: Beam element.

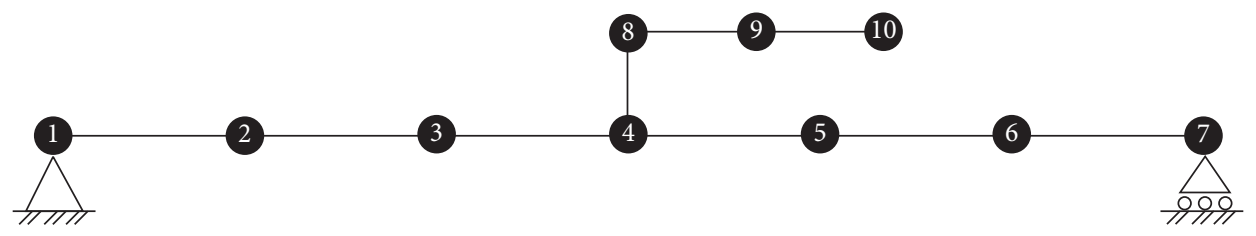

Figure 7: The FE model of the pipeline structure with the beam DVA.

a pipeline, by which 12 coupled differential equations are analyzed. All geometric characteristics and physical properties of the pipeline and the amplitude and frequency of the sinusoidal force are defined as program inputs by means of block diagrams. Finally, the "displacement of the midpoint of the pipe to the time" is plotted. In the second part of the simulation, the 16 extracted equations are analyzed and the displacement of the midpoint of the pipe is plotted and compared with the first case. The ODE45 variable-step solver is used in model analysis.

The accuracy of the FEM model can be improved by further nodes and elements; however, this may reduce the computational efficiency. Several models with a different number of elements were investigated. This section compares the FEM pipeline model without the beam DVA model with analytical beam motion equations in the Euler-Bernoulli theory on the simple support applied by a centralized force in the middle of the beam. The transverse vibration motion of the beams is in the form of a fourthorder partial differential equation as follows:

$$
\begin{aligned}
& E_{p} I_{p} \frac{\partial^{4} x_{p}}{\partial x^{4}}(x, t)+\rho_{p} A_{p} \frac{\partial^{4} x_{p}}{\partial x^{4}}(x, t)=f(x, t), \\
& x_{p}(x, t)=\frac{2 F_{0}}{\rho_{p} A_{p} L_{p}} \sum_{n=1}^{\infty} \frac{1}{\omega_{n_{p}}^{2}-\omega^{2}} \sin ^{2} \frac{n \pi}{2} \sin \omega t .
\end{aligned}
$$

The response of the pipeline structure using the mode superposition principle is

Figure 8 shows the displacement of the midpoint of the 1-inch pipeline structure with respect to time and an excitation frequency of $22 \mathrm{~Hz}$, using equation (12) and simulating the FE model. As can be seen, there is an acceptable match.

\section{Comparison of Experimental Study: Finite Element Model with DA}

To establish the amplitude ratio of the pipeline without and with the beam DVA, three cases were analyzed using 


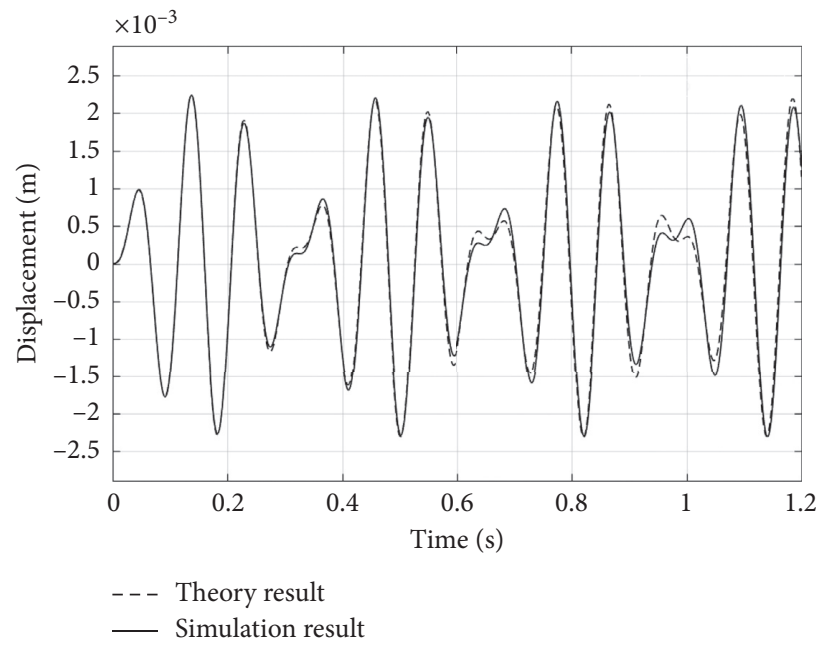

FIgURE 8: Comparison between theoretical and FE results without beam DVA, 1-inch pipeline, with an excitation frequency of $22 \mathrm{~Hz}$.

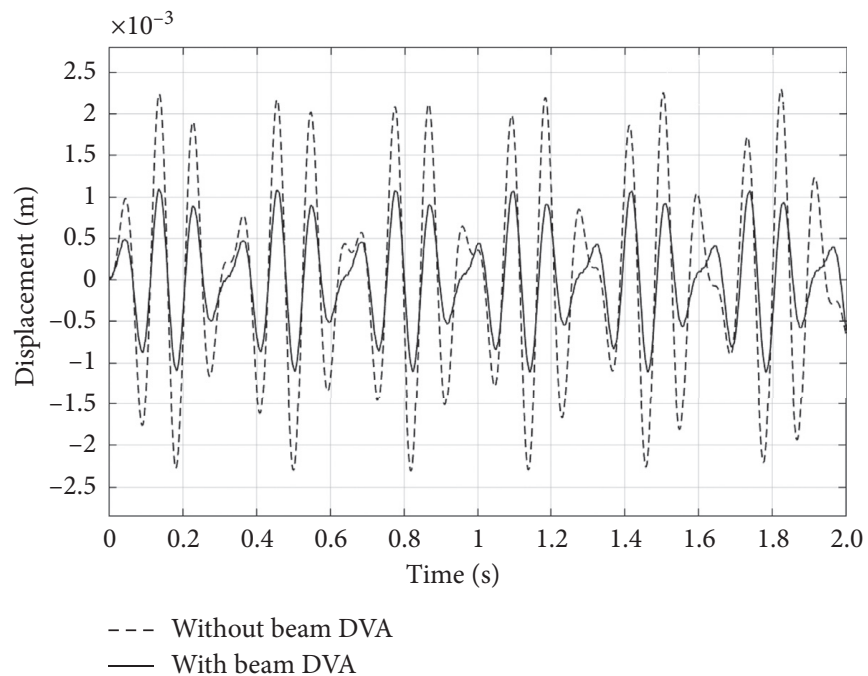

Figure 9: Numerical results of the 1-inch pipeline structure under the excitation frequency of $12.5 \mathrm{~Hz}$.

experimental results, numerical results, and methodology described in this paper. In each case, the beam DVA with the same specification was designed for comparison.

6.1. Case 1. In the 1-inch pipe with the specifications listed in Table 1.

Beam DVA: threaded rod M8 with length of $0.18 \mathrm{~m}$ and mass of $2.35 \mathrm{~kg}$.

Frequency of exciting force of $12.5 \mathrm{~Hz}$.

Numerical and experimental results are presented in Figures 9 and 10. As shown in the figures, the amplitude ratios of the pipeline $\left(x_{\mathrm{pa}} / x_{p}\right)$ are 0.475 and 0.397 , respectively. Also, according to equation (8), $\pi_{S}=0.3814$.

6.2. Case 2. In the 2-inch pipe with the specifications listed in Table 1.

Beam DVA: threaded rod M16 with length of $0.245 \mathrm{~m}$ and mass of $10 \mathrm{~kg}$.

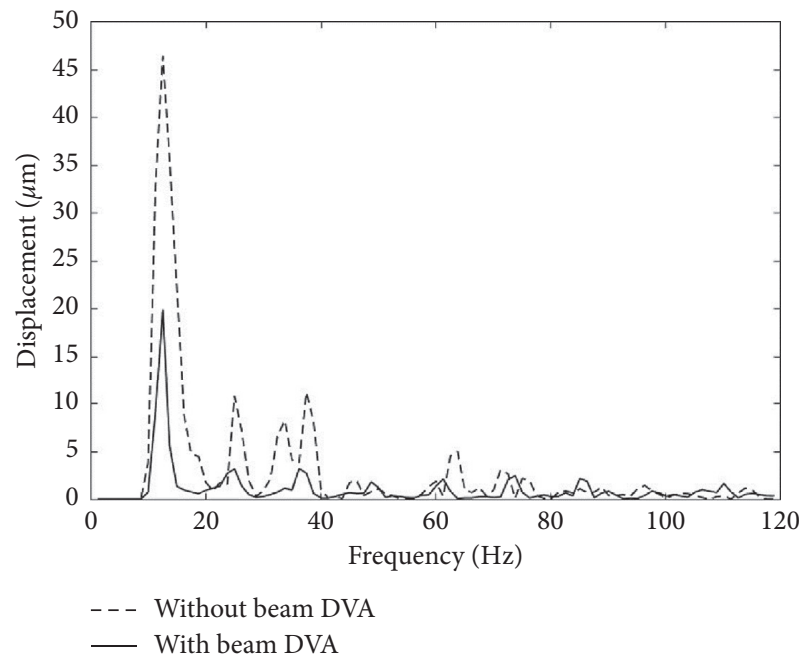

FIGURE 10: Experimental results of the 1-inch pipeline structure with the inverter operating at around $12.5 \mathrm{~Hz}$. 


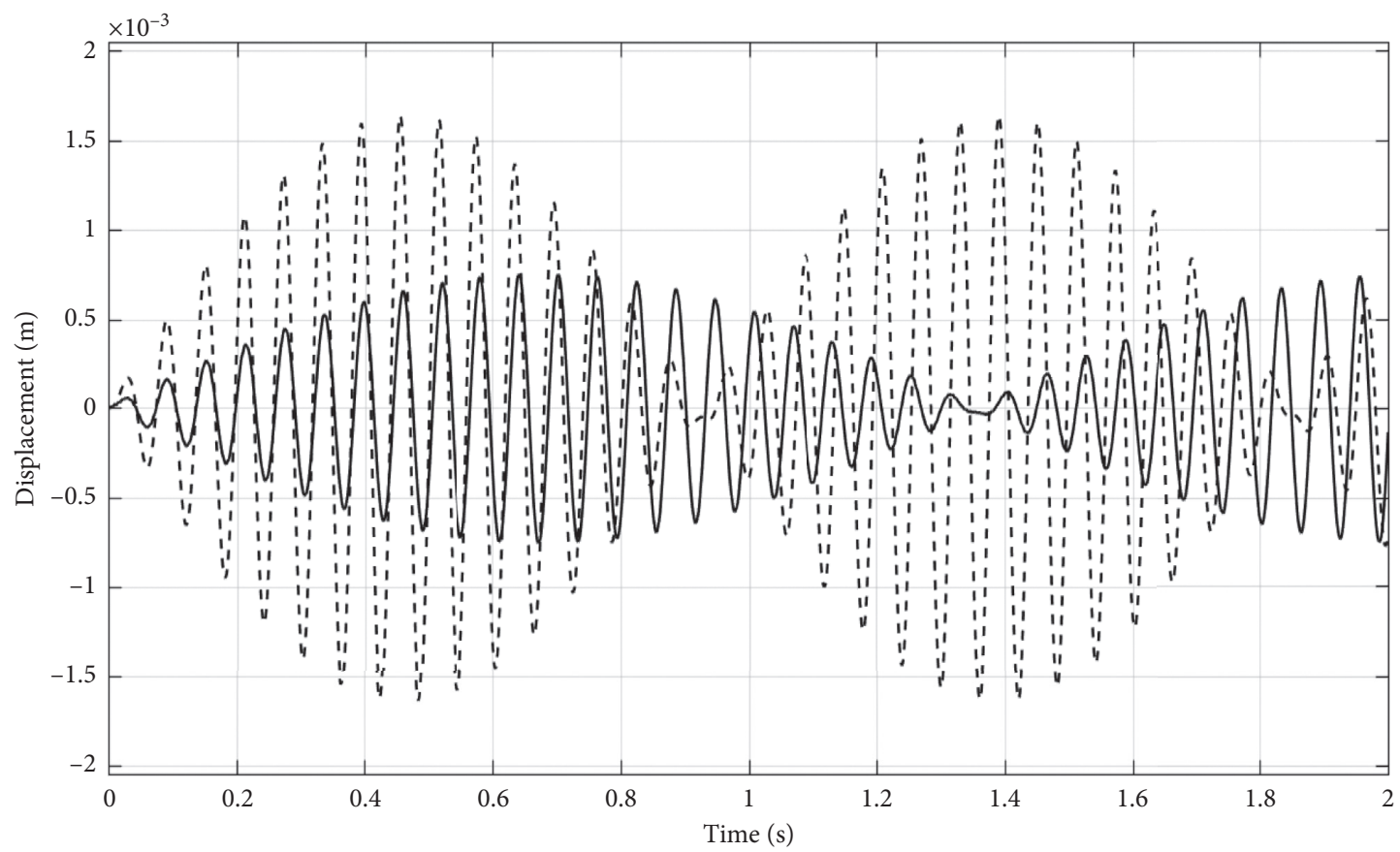

- - - Without beam DVA

_ With beam DVA

FIgURE 11: Numerical results of the 2-inch pipeline structure under the excitation frequency of $16 \mathrm{~Hz}$.

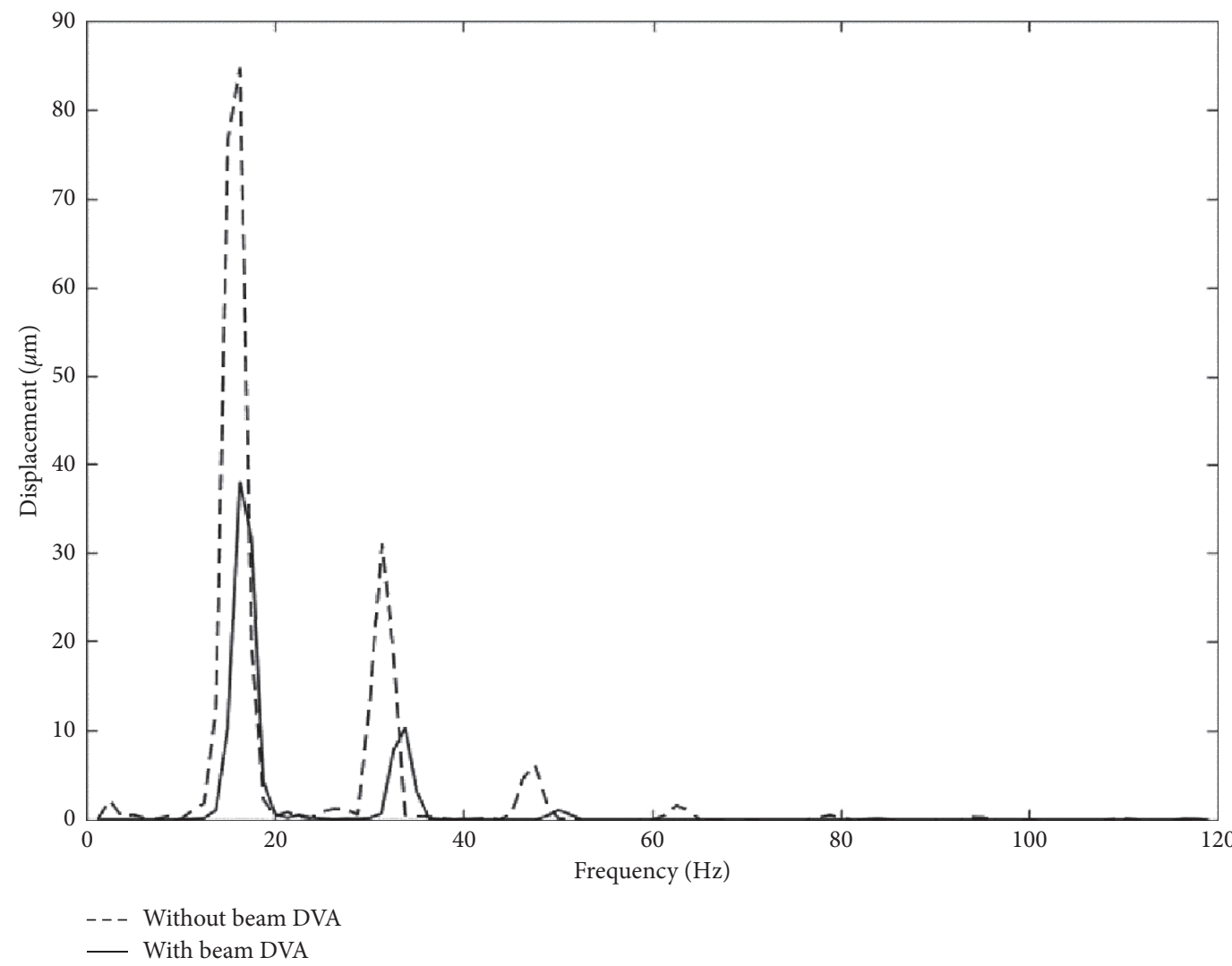

FIgURE 12: Experimental results of the 2-inch pipeline structure with the inverter operating at around $16 \mathrm{~Hz}$. 


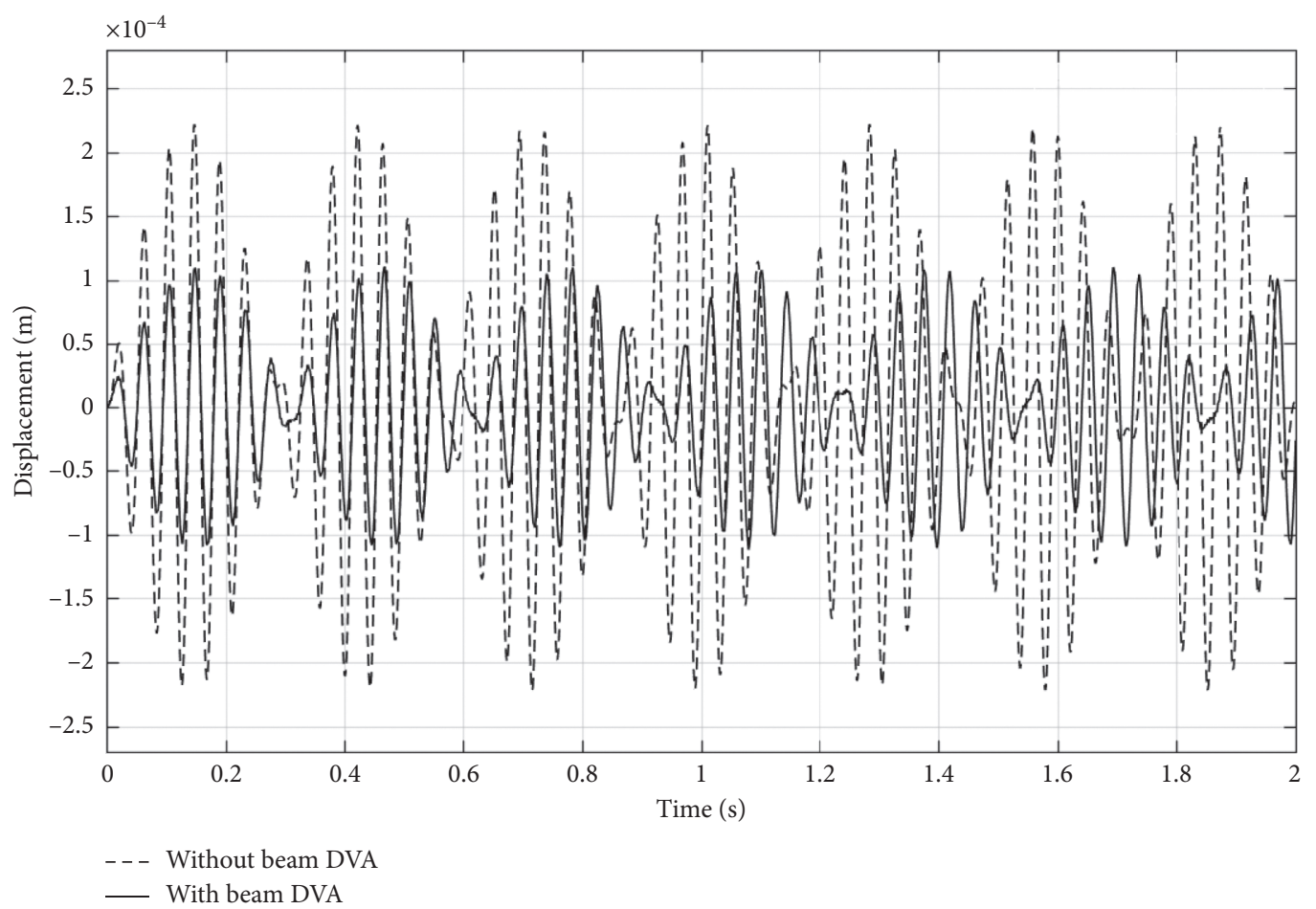

FIgURE 13: Numerical results of the 3 -inch pipeline structure under the excitation frequency of $22 \mathrm{~Hz}$.

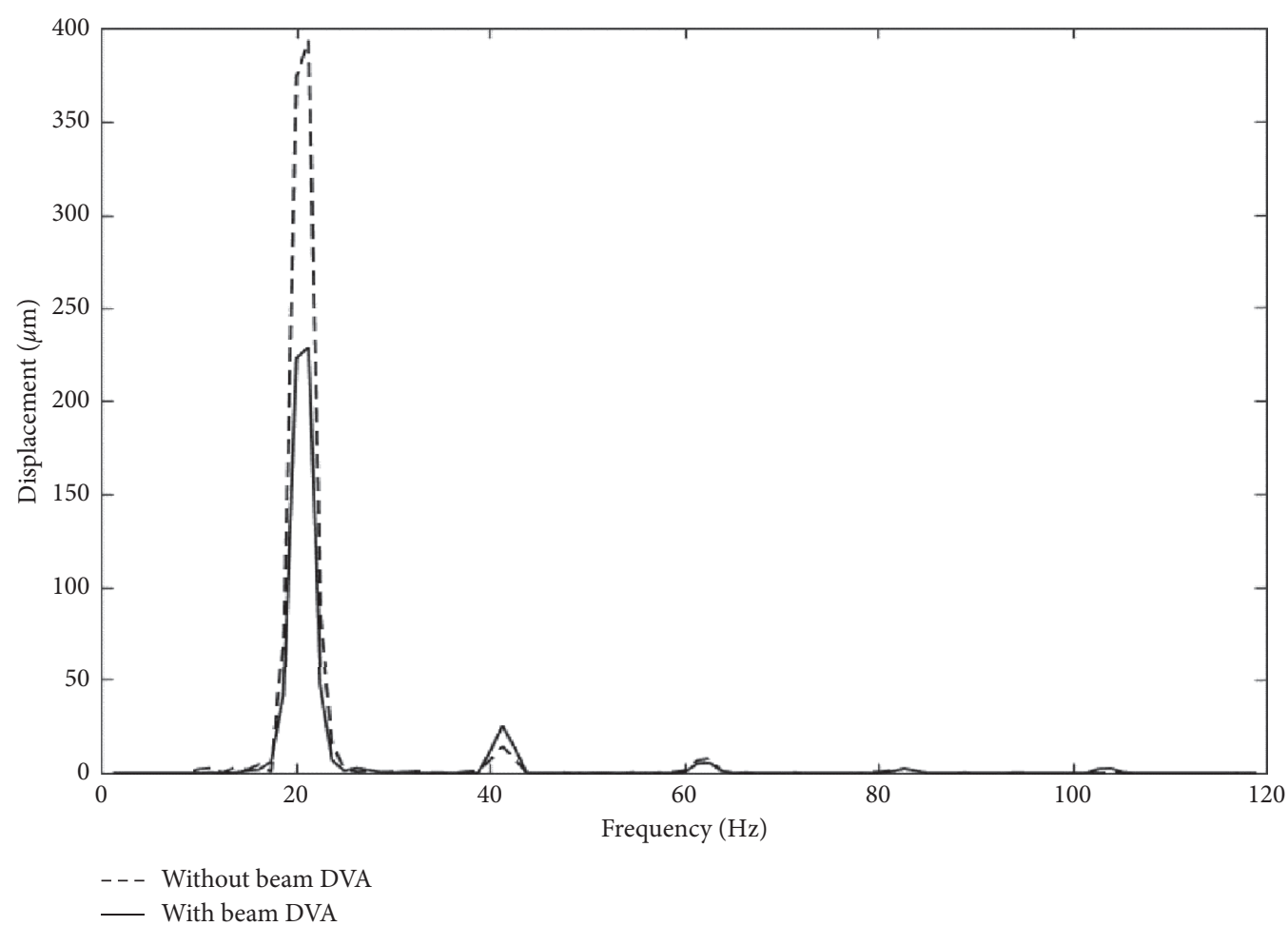

FIGURE 14: Experimental results of the 3-inch pipeline structure with the inverter operating at around $22 \mathrm{~Hz}$.

Frequency of exciting force of $16 \mathrm{~Hz}$.

Numerical and experimental results are presented in Figures 11 and 12. As shown in the figures, the amplitude ratios of the pipeline $\left(x_{\mathrm{pa}} / x_{p}\right)$ are 0.457 and 0.427 , respectively. Also, according to Equation (8), $\pi_{S}=0.401$.
6.3. Case 3. In the 3-inch pipe with the specifications listed in Table 1.

Beam DVA: threaded rod M16 with length of $0.19 \mathrm{~m}$ and massof $10 \mathrm{~kg}$.

Frequency of exciting force of $22 \mathrm{~Hz}$. 
Numerical and experimental results are presented in Figures 13 and 14. As shown in the figures, the amplitude ratios of the pipeline $\left(x_{\mathrm{pa}} / x_{p}\right)$ are 0.499 and 0.56 , respectively. Also, according to equation (8), $\pi_{S}=0.499$.

As can be seen, the proposed method agree quite well with experimental, numerical, and DA results. Also, there are some errors between the numerical and experimental results. These differences can referred to the restrictions of the motor.

\section{Conclusions}

In this paper, the beam DVA was proposed to reduce the vibration of the pipeline structures. For this purpose, dimensional analysis technique was developed for the prediction of the amplitude ratio of the pipeline without and with the beam DVA. Using the dimensional analysis technique, a new, simple, and reliable method was introduced to design dynamic vibration absorbers for pipeline structures. Buckingham's theory in dimensional analysis makes it possible to identify nine dimensionless groups. In the experimental study, a beam DVA was installed on 1-inch, 2inch, and 3-inch pipes, and harmonic force was applied to the pipeline. Using the results obtained from the experimental study and applying the multiple linear regression method, new correlation was derived for designing optimal beam DVA. Also, the results obtained are validated with FEM and indicated that the method based on DA proposed is a good alternative solution for the quick determination of the amplitude ratio of the pipeline structure without excessive computational costs or user expertise.

In addition, nondimensionalization of an equation allows the identification of significant and insignificant terms, and this is very effective in the design of beam DVA. The mass ratio has the greatest effect on amplitude ratio of the pipeline and stiffness ratio has the lowest effect.

\section{Data Availability}

The data used support the findings of this study are available from the corresponding author upon reasonable request.

\section{Conflicts of Interest}

The authors declare that there are no conflicts of interest regarding the publication of this paper.

\section{References}

[1] M. A. Norris, K. R. Ptak, B. A. Zamora, and J. D. Hart, "Implementation of tuned vibration absorbers for above ground pipeline vibration control," in Proceedings of International Pipeline Conference, American Society of Mechanical Engineers (ASME), New York, NY, USA, October 2000.

[2] K. Nabeel, A. Al-Sahib, A. N. Jameel, and O. Abdulateef, "Investigation into the vibration characteristics and stability of a welded pipe conveying fluid," Jordan Journal of $\mathrm{Me}$ chanical and Industrial Engineering, vol. 4, pp. 378-387, 2010.

[3] A. Senalp, A. Arikoglu, I. Ozkol, and V. Ziya Dogan, "Dynamic response of a finite length euler-bernoulli beam on linear and nonlinear viscoelastic foundations to a concentrated moving force," Journal of Mechanical Science and Technology, vol. 24, no. 10, pp. 1957-1961, 2010.

[4] Ç. Demir and Ö. Civalek, "A new nonlocal FEM via hermitian cubic shape functions for thermal vibration of nano beams surrounded by an elastic matrix," Composite Structures, vol. 168, pp. 872-884, 2017.

[5] W. Latas., "Multiple tuned tunable translational-rotational vibration absorbers in beam," Vibrations in Physical Systems, vol. 26, pp. 145-152, 2014.

[6] Ö. Civalek, "Free vibration and buckling analyses of composite plates with straight-sided quadrilateral domain based on DSC approach," Finite Elements in Analysis and Design, vol. 43, no. 13, pp. 1013-1022, 2007.

[7] A. M. R Syaiful, I. Zaman, B. Manshoor, A. Khalid, S. W. Chan, and M. S. Mohd Sani, "Theoretical modelling of a beam with attached spring-mass-damper system," MATEC Web of Conference, vol. 90, p. 01030, 2017.

[8] M. H. Zainulabidin, N. A. Mat Jusoh, N. Jaini, and A. S. M. Kassim, "Effect of tuned absorber location on beam structure vibration by finite element analysis," ARPN Journal of Engineering and Applied Sciences, vol. 11, pp. 8585-8591, 2016.

[9] S. Sutar, R. Madabhushi, and R. B. Poosa, "Finite element analysis of piping vibration with guided supports," Journal of Mechanical Engineering and Automation, vol. 3, pp. 96-106, 2016.

[10] G. B. Song, P. Zhang, L. Y. Li et al., "Vibration control of a pipeline structure using pounding tuned mass damper," American Society of Civil Engineers (ASCE), vol. 142, no. 6, Article ID 04016031, 2016.

[11] K. S. Fong and A. Y. Mohd Yassin, "Fluid-structure interaction (FSI) of damped oil conveying pipeline system by finite element method," MATEC Web Of Conference, vol. 111, p. 01005, 2017.

[12] S. Chang, W. Sun, G. C. Sung, and D. Kim, "Vibration control of nuclear power plant piping system using stockbridge damper under earthquakes," Hindawi Publishing Corporation Science and Technology of Nuclear Installations, p. 12, Article ID 5014093, 2016.

[13] Y. Hua, W. Wong, and L. Cheng, "Optimal design of a beambased dynamic vibration absorber using fixed-points theory," Journal of Sound and Vibration, vol. 421, pp. 111-131, 2018.

[14] Y. H. Tyng, O. Z. Chao, K. K. Kuan, Z. Ismail, A. G. Abdul Rahman, and C. W. Tong, "Similitude study of an in-service industrial piping system under high flow induced vibration," Journal of Mechanical Science and Technology, vol. 31, no. 8, pp. 3705-3713, 2018.

[15] G. M. Reddy and V. D. Reddy, "Theoretical investigations on dimensional analysis of ball bearing parameters by using Buckingham pi-theorem," Procedia Engineering, vol. 97, pp. 1305-1311, 2014.

[16] K. V. Allamraju and K. Srikanth, "Dimensional analysis of PZT-5H transducers," Materials Today: Proceedings, vol. 4, no. 2, pp. 2384-2389, 2017.

[17] S. Hassani, R. Saidur, S. Mekhilef, and A. Hepbasli, "A new correlation for predicting the thermal conductivity of nanofluids; using dimensional analysis," International Journal of Heat and Mass Transfer, vol. 90, pp. 121-130, 2015.

[18] P. S. Thakare, S. M. Salodkar, and C. C. Handa, "Development of mathematical model for top roller displacement of threeroller bending machine using dimensional analysis," in Proceedings of International Conference on Intelligent Manufacturing and Automatio, pp. 125-132, Springer, Singapore, Singapore, 2019. 